\title{
Towards Service and Networking Intelligence for Humanity: A Report on APNOMS 2020
}

\author{
Seungjoon Seok ${ }^{1}$ (D) Y Yuncheng Zhu ${ }^{2}$. Jen-Jee Chen ${ }^{3} \cdot$ Woojin Seok ${ }^{1,6}$. \\ Kazuhiko Kinoshita ${ }^{4} \cdot$ Jiun-Long Huang ${ }^{3} \cdot$ Hongtaek Ju $^{5}$
}

Received: 7 December 2020 / Revised: 2 March 2021 / Accepted: 4 March 2021 /

Published online: 14 June 2021

(C) The Author(s), under exclusive licence to Springer Science+Business Media, LLC, part of Springer Nature 2021

\section{Abstract}

This article presents a report on APNOMS 2020, which was held on September 22-25, 2020, at EXCO, Daegu, South Korea. The theme of APNOMS 2020 was "Towards Service and Networking Intelligence for Humanity."

Keywords Intelligent network operations and management - IoT and wireless sensor networks · Container and cloud computing · Networked applications services · Edge computing

Seungjoon Seok

sjseok@kyungnam.ac.kr

Jen-Jee Chen

jenjee@nctu.edu.tw

Woojin Seok

wjseok@gmail.com

Kazuhiko Kinoshita

kazuhiko@is.tokushima-u.ac.jp

Jiun-Long Huang

jlhuang@cs.nctu.edu.tw

Hongtaek Ju

juht@kmu.ac.kr

1 Kyungnam University, Changwon, Korea

2 Google, Minato, Japan

3 National Chiao Tung University, Hsinchu, Taiwan

4 Tokushima University, Tokushima, Japan

5 Keimyung University, Daegu, Korea

6 Korea Institute of Science and Technology Information, Daejeon, Korea 


\section{Introduction}

APNOMS (Asia-Pacific Network Operations and Management Symposium) has been a premier conference on network operations and management in the AsiaPacific region. The symposium has been organizing the researchers' congregation since 1997 to exchange knowledge and ideas. The 21st APNOMS 2020 (www. apnoms.org/2020/) was held on September 22-25, 2020. Due to the COVID-19 pandemic, APNOMS 2020 was converted into a hybrid conference. The online part of it was held via the Zoom Webinar event platform, while the off-line part of it was held at the famous Exhibition and Convention Center In Korea, EXCO, Daegu, South Korea. APNOMS 2020 was organized by the Institute of Electronics, Information and Communication Engineers (IEICE) Technical Committee on Information Communication Management (ICM) and the Korea Information and Communications Society (KICS) Technical Committee on Korean Network Operations and Management (KNOM) with support from IEEE ComSoc (IEEE Communications Society), DAEGU Convention \& Visitors Bureau (dcvb), Chunghwa Telecom, BcMon (Blockchain Monitoring System), BOSCO Technologies and Ciena.

APNOMS plays a crucial role in exchanging and discussing all aspects of operations and management of telecommunications networks, enterprise networks, the internet, and their services among the academic community, vendors, and the telecommunication industry at large Asia-Pacific region. As in the previous APNOMS symposia [1-17], APNOMS 2020 was a great success. It attracted nearly 130 scholars, researchers, policymakers, practitioners, service providers, and vendors from 12 countries.

With the evolution of networking technologies and artificial intelligence, the world is going through a rapid change. The new technologies' advantages should be extended to all: literate and illiterate, non-disabled and disabled users, urban and rural people. In this respect, our world, in fact, is divided into two: privileged and underprivileged. There is a need to bridge the gap between underprivileged users and cyber society. With this aim in our mind, we have chosen the theme of "Towards Service and Networking Intelligence for Humanity" for this year's symposium. APNOMS 2020 focused on the new trend of Artificial Intelligence, Networking Intelligence, 5G networks, and digital services, which enable flexible and dynamic system management for humans. Discussions at the conference showed how new technologies (i.e., Blockchain, AI, IoT, VR, and 5G) would lead to one of our lifetime's most significant technological conversions, with endless possibilities.

APNOMS 2020 prepared an excellent 4-full day program with keynotes, tutorials, technical sessions, poster sessions, innovation sessions, special sessions, exhibitions, and an interactive Distinguished Experts Panel (DEP). Synopses of each event are given in the following sections. The summarized events show the entire scenario of APNOMS 2020 and current research trends on Service and Networking Intelligence for Humanity. 


\section{Technical, Poster, Innovation, and Demonstration Sessions}

The symposium's main body consisted of ten technical sessions, four-poster sessions, including two innovation sessions. This year, we received 119 submissions for technical sessions and poster sessions from 12 different countries. We selected 40 technical papers for oral sessions; unfortunately, two papers were withdrawn before the conference. The acceptance rate for oral presentation was $39.17 \%$. For reference, last year, 127 papers were submitted, and the acceptance rate for oral presentation was $29.03 \%$ (35 papers in 9 technical sessions). Similar to previous years, for each paper, we provided at least three independent reviews, TPC members, across one round.

Accepted papers covered a wide range of essential and latest research and development results in smart networks and services' operations and management. The ten technical sessions covered research areas including Intelligent Network Management, TCP/IP and SDN, Internet of Things and Wireless Sensor Networks, Mobile and Wireless Network, Network Security, Network Monitoring and Measurements, Networked Applications Services including Blockchain technologies, and Edge Computing. This year, 5G Network Communication and Cyber-Physical Security areas were the most attractive. The average number of webinar attendees in technical sessions was equal to 24.5. In comparison, the average number of the audience (on-site attendees) was equal to 12. Technical session 2 "TCP/IP and SDN" attracted the most participants (32 webinar attendees and 15 on-site attendees).

This year, we expanded the poster sessions and held four sessions in total. 50 poster presentations were held in the symposium, and each poster session presented essential and exciting topics. As we held the four-poster sessions online (without allowing on-site participation), the average number of webinar attendees in poster sessions was equal to 30.5. Poster session 3 attracted most of the participants (36 webinar attendees). The interested attendees to the poster presentations asked many questions to gather more information and make the sessions enjoyable.

The Innovation Sessions were also organized to present and discuss ongoing research, work-in-progress ideas, practical solutions, experimental studies, and various topics of interest to the community. Nine papers were presented in two innovation sessions focusing on "Network Operations \& Management" and "Service \& Networking Intelligence" topics. The acceptance rate for innovations papers was $100 \%$. Ten papers were selected (unfortunately, one paper was withdrawn before the conference). The average number of webinar attendees in innovations sessions was equal to 23.5, while the average number of the audience (on-site attendees) was equal to 10. Innovation session 1 "Network Operations and Management" attracted the most participants (31 webinar attendees and 17 on-site attendees).

The steadfast effort of the TPC co-chairs Seungjoon Seok (Kyungnam University, Korea), Yuncheng Zhu (Google, Japan), and Jen-Jee Chen (National Chiao Tung University, Taiwan) along with other 94 PC members enabled us to meet the deadline to notify authors. 


\section{Tutorials}

To provide the participates in the tutorial sessions an excellent opportunity for lifelong learning. Tutorial Co-Chairs Sangheon Pack (Korea University, Korea), Noriaki Kamiyama (Fukuoka University, Japan), and Chi-Yu Li (National Chiao Tung University, Taiwan) selected the most suitable tutorials that match the APNOMS 2020 conference theme "Towards Service and Networking Intelligence for Humanity" from all the submitted Tutorial proposals.

Four tutorial sessions in two parallel tracks covering the latest hot topics were held on the second day of the symposium. In the first two tutorial sessions, Mr. Kyung-Joon Park (DGIST, South Korea) and Mr. Tze-Jie Tan (National Chiao Tung Univ., Taiwan) gave tutorials on "Cyber-Physical Security - A Networking Perspective" and "From 5G Network Architecture to 5G Core Network OpenSource Project (Free5G)" respectively. The tutorials were given simultaneously in two different Zoom Webinar rooms. The tutorial given by Mr. Kyung-Joon Park investigated several attack and security issues in the Cyber-Physical Security (CPS) as the network control system and discussed possible solutions to these issues by introducing a resilient SDN framework against pole dynamics attack. The tutorial given by Mr. Tze-Jie Tan provided details on 4G and 5G core network and operations, internetworking between $4 \mathrm{G}$ and $5 \mathrm{G}$ systems, network slice management, $5 \mathrm{G}$ deployment strategies, and expose some of the $5 \mathrm{G}$ open-source projects such as Free5GC.

In the second two tutorial sessions, Prof. Shibo He (Zhejiang Univ., China) gave a tutorial on "Long-range Low-power Localization for the Internet of Things.“ While Prof. Kazuya Tsukamoto (Kyushu Institute of Technology, Japan) gave a tutorial on "Geolocation-centric Information Platform for Resilient Spatiotemporal Service Management". Prof. Shibo He introduced a new approach for the localization of IoT devices based on a long-range low-power (LoRa) wireless communication protocol which enables the outdoor and indoor localization of connected devices. Prof. Kazuya Tsukamoto introduced the SDGs and Society 5.0, advantages and major challenges, and presented the critical player, concept, and essential functions for the Geolocation-centric information platform (GCIP). The four tutorials attracted many audiences and generated discussions on these topics regardless that the presentations were held online. The average number of webinar attendees in tutorial sessions was equal to 22 , while the average number of the audience (on-site attendees) was equal to 10.

\section{Keynotes}

Three keynote speakers shared their visions and perspectives on 5G Deployment and Artificial intelligence in 5G technology during this symposium.

On the second day, Prof. Jyh-Cheng Chen (National Chiao Tung Univ., Taiwan) delivered a keynote on "Free 5GC - Free the Cellular Core Network," 
highlighting Taiwan's current efforts and long term plans for 5th generation (5G) mobile core networks. He also mentioned that this project's ultimate goal is to implement the 5G core network (5GC) defined in 3GPP Release 15 (R15) and beyond.

On the third day, Dr. Hiroaki Harai (National Institute of Information and Communications Technology, Japan) gave a keynote on "AI-Support Network Control and Management towards Beyond 5G Era". He explained the possibility of applying machine learning to service network platform control and End-to-End network operation for $5 \mathrm{G}$ Era.

On the last day, Dr. Lee Young Ro (National Information Society Agency, South Korea) delivered a speech on " $5 \mathrm{G}+$ MEC Deployment for the Public Administration". He introduced $5 \mathrm{G}$ progress in South Korea and presented on the flagship projects to promote $5 \mathrm{G}$ in the country.

\section{Special Session}

Two special sessions were held on the third and fourth day of the symposium. Seven representatives of various countries from Asia-Pacific region discussed the latest research topics on Multi-access Edge Computing (MEC)-5G and Beyond, Government Policies, and Standardization Activities on Telecommunication.

On the third day, four speakers gave talks on "Multi-access Edge Computing (MEC) - 5G and Beyond" as the current hottest issues. Dr. Sangho Shin (SK Telecom, Korea) gave a talk on "Journey to the world's first 5G MEC platform." He introduced cloud computing in a $5 \mathrm{G}$ network edge. He spoke about the deployment of $5 \mathrm{G}$ in South Korea for the nationwide-services or built on-premises to provide enterprise-specific services, working with CPS to expand the edge ecosystem. Mr. Kibeom Park (Samsung Electronics, Korea) delivered a speech on "E2E 5G Network Automation (Network Slicing/Edge Cloud/SDN)". He introduced the needs for network slicing in 5G, network slicing trends, and use cases in South Korea. Mr. Kibeom Park also explained how SAMSUNG is actively participating in the 5G network open source and open standard. Besides, he provided an E2E network slicing architecture based on the O-RAN/3GPP/ETSI standard and critical solution in network slicing design from their inventions to potential future research. Dr. Ling-Jyh Chen (Academia Sinica, Taiwan) gave a speech on "Making Sense of the Internet of Things: using AirBox as an Example." Dr. Ling-Jyh Chen gave an overview of the global impacts of the IoT industry on environmental governance. He also introduced the AirBox project and highlighted how the open-source project helps make systems more scalable. Mr. Shinsaku Akiyama (DOCOMO Technology, Japan) provided a talk on "The Road to Zero Touch Operation." He introduced network management in both $3 \mathrm{G}$ and $4 \mathrm{G}$ Era and later compared it with the $5 \mathrm{G}$ network management, mainly network slicing. Further, he instructed the DOCOMO's architecture for Zero-touch operation and potential future research.

On the last day, Three speakers gave talks on "Government Policies and Standardization Activities on Telecommunication." Dr. Aris Risdianto (ONF (Open Networking Foundation) Ambassador, Singapore) gave a talk on "COMAC (Converged 
Multi-access and Core) Project." He presented CORD with the new network edge platform. Then he introduced the COMAC platform for converging 5G and LTE mobile networks together with broadband access and core network. Mr. Doan Quang Hoan (Secretary-general of the Radio Electronics Association of Vietnam (REV), Vietnam) gave a speech on "Deploy 5G Networks Effectively and Safely". He gave an overview of the significant differences between $4 \mathrm{G}$ and 5G. He introduced the $5 \mathrm{G}$ implementation challenges and major policy issues in Vietnam as an example. He further emphasized that $4 \mathrm{G}$ will still be a primary for the next five years. Prof. Teerapat Sanguankotchakorn (Asian Institute of Technology, Thailand) provided a talk on "5G Development and Deployment in Thailand". He talked about the 5G roadmap and spectrum auction and the current mobile coverage area in Thailand. He provided some issues related to the $5 \mathrm{G}$ frequency spectrum allocation. He further presented the achievements of Thailand in 5G development.

\section{Exhibitions}

The exhibitions program provided vendors and service providers an opportunity to exhibit their latest technologies over four days. Four exhibitors, including BcMon (South Korea), Chunghwa Telecom (Taiwan), BOSCO Technologies (Japan), and Ciena (South Korea), participated in the exhibition program. Since the conference took place remotely, we asked each of our exhibitors to provide us with a promoting video of $5 \mathrm{~min}$. We displayed the exhibitors' videos in a row during the breaks. Each exhibition attracted many attendees, lively discussions by all attendees were conducted.

BcMon (Blockchain Monitoring System) reveals and logs all blocks/transactions on public and permissioned blockchains. The information collected by the BcMon includes blocks/transaction data, mempool data, nodes data, used program, network data. BcMon also gives an overview of multiple chains, mainly Bitcoin and Ethereum. Using intuitive dashboards, $\mathrm{BcM}$ Mon provides a profound understanding of blockchain components.

Chunghwa Telecom presented 5G Network E2E Service Assurance, an integrated monitoring system that provides intelligent obstacle locations for end-to-end circuits across multiple domains. This system shows the 5G Crosshaul service's operating status composed of the base station, Mobile Fronthaul (MFH), Mobile Backhaul $(\mathrm{MBH})$, and core network. The integrated and regularized data of service status, abnormal events of network equipment, and circuits from different network domains establish a single monitor panel. Besides, Their system provides a notification mechanism to notify relevant personnel proactively and shows root cause analysis results.

BOSCO Technologies dealt with its IT management solution for the IoT era. Being motivated by extensive ICT infrastructure and complicated IoT and SDN/ NFV, BOSCO Technologies is developing unified, simplified, and flexible ICT infrastructure management that does not depend on other network environments. BOSCO provides a SMART-GW, the brand-new web-based unified and simplified ICT management system where an operator can log in to SMART-GW via a web browser and establish a connection, such as SSH, HTTP, RDP, and so on to 
manage the ICT infrastructures where all the connection behaviors are logged and traceable with user-friendly dashboards.

Ciena New paradigm and advanced technology in 5G \& ESL help out customers to migrate from $4 \mathrm{G}$ to $5 \mathrm{G}$ with minimal risk and the ability to rapidly generate a greater return on their investments. To ensure connectivity Anywhere and Anytime, to Anyone and Anything and bring new QoS services, the company leverages intelligent automation, next-generation routing platforms and advanced professional services to support the delivery of differentiated 5G services, such as eMBB (enhanced Mobile Broadband), mMTC (massive Machine-Type Communications) and urLLC (ultra-reliable Low-Latency Communications).

\section{Distinguished Experts Panel}

The APNOMS 2020 DEP session was led by the Session Chair Prof. Yoonhee Kim (Sookmyung Woman's Univ., Korea). The participating panelists from Korea, Taiwan, and Japan first made presentations, followed by a Q\&A session.

The DEP Korea panelist Prof. Ji-Woong Choi (DGIST, South Korea) gave a talk on "Future high-speed in-vehicle networks for connected and autonomous driving." He initiated his speech by explaining the IVNs (in-vehicle networks) technologies. Then, he introduced how the deployment of the new IVNs technologies will improve autonomous driving performance by supporting high-speed and low-latency communications among numerous sensors and controllers.

The DEP Taiwan panelist Prof. Shiao-Li Tsao (National Chiao Tung Univ., Taiwan) provided a speech on "The last mile of AI applications and services." He struck up his talk by introducing the industrial AI applications and services, explaining their difficulties and the last mile for industrial AI applications and services deployment. Finally, he shared some of his viewpoints and provided some possible solutions for that issue.

The DEP Japan panelist Dr. Takayuki Kuroda (NEC Corporation, Japan), gave a talk on "Introduction about the automation of intent-based network designing with machine learning." He initiated his speech by introducing automated network operations and implementation challenges. Then, he introduced a new network operation automation method that utilizes the intent-based network design method and AI technology.

The DEP Korea panelist Dr. Taesang Choi (ETRI, South Korea), delivered a presentation on " $6 \mathrm{G}$ in the Sky: On-Demand Intelligence at the Edge of 3D Networks". In this talk, Dr. Taesang Choi provided an overview of the 6G technology and Mobile Edge Computing (MEC). Then, He gave an example of 6G architecture by explaining the complexity of the overall network.

The panelists and attendees had a fruitful discussion, focusing on "what is most necessary for supporting network intelligence technologies" and "what is needed most urgently to enable optimized 5G mobile communications network management and the most critical security issue." 


\section{APNOMS 2019 Awards}

The APNOMS 2020 organizing committee selected the top four papers presented in the technical session for the "Best Paper Award." The Best Paper Award Committee was organized within the organizing committee with TPC co-chairs as core members. Before the symposium, 12 papers were nominated for the best paper consideration from four countries. The award committee evaluated the nominees' presentations and finally selected four papers with the highest overall (paper and presentation) scores. The selected papers were "Analysis of Ethereum Network Properties and Behavior of Influential Nodes, " by Soo Hoon Maeng (Keimyung Univ., South Korea), Meryam Essaid (Keimyung Univ., South Korea), Hong Taek Ju (Keimyung Univ., South Korea), “A Design of Port Scan Detection Method Based on the Characteristics of Packet-In Messages in OpenFlow Networks," by Daichi Ono (Tohoku Univ., Japan), Satoru Izumi (National Institute of Technology, Sendai College, Japan), Toru Abe (Tohoku Univ., Japan), Takuo Suganuma (Tohoku Univ., Japan), "Improving Scanner Data Collection in P4-based SDN ," by Yun-Zhan Cai (National Cheng Kung Univ., Taiwan), Chih-Hao Lai (National Cheng Kung Univ., Taiwan), Yu-Ting Wang (National Cheng Kung Univ., Taiwan), Meng-Hsun Tsai (National Cheng Kung Univ., Taiwan), and "Entropy-based IoT Devices Identification," by Hung Nguyen-An (Hanoi Univ. of Science and Technology, Vietnam \& Shibaura Institute of Technology, Japan), Thomas Silverston (Shibaura Institute of Technology, Japan), Taku Yamazaki (Shibaura Institute of Technology, Japan), Takumi Miyoshi (Shibaura Institute of Technology, Japan).

The Best Paper Award committee also selected four "Student Best Paper Award" from the student's speaker in the technical session. This award was given to students who have made excellent presentations and papers from the technical session speakers. The awarded persons were Heegon Kim (POSTECH, South Korea) for the paper entitled "Graph Neural Network-based Virtual Network Function Management,“" Tomoya Tanaka (Kobe Univ., Japan) for the paper entitled "Topic-based Allocation of Distributed Message Processors on EdgeServers for Real-time Notification Service," Yi-Chia Chen (National Chiao Tung Univ., Taiwan) for the paper entitled "Distributed Profitable Deployment of Network Services to Geo-distributed Edge Systems," and Xu Zhang (Sun Yat-Sen Univ., China) for the paper entitled "Deep Multi-Agent Reinforcement Learning for Resource Allocation in D2D Communication Underlying Cellular Networks."

\section{Concluding Remarks}

APNOMS 2020 paid attention to several exciting and essential topics, such as SDN and NFV/VNF management, edge and IoT management, and network resource management. APNOMS 2020 was a very successful symposium. It was 
well attended, and the feedback on all aspects of the symposium program was very positive. It contributed to the growth of APNOMS into a critical international symposium. The attendees feedback reinforced the positive aspects of the symposium: well-mixed participation from both industry and academia in technical contributions, the tradition of special sessions focusing on experiences and lessons learned by different countries in the Asia-Pacific region, excellent venue and social events, and the overall collaborative, interactive and friendly atmosphere of the symposium.

In APNOMS 2020, the technical and poster session papers were published in IEEE Xplore like previous APNOMS. Also, the proceedings and innovation session papers have been distributed to the participants from the symposium website. Most of the presentation files (keynotes, tutorials, special sessions, and DEP) are all available on the symposium website: . The APNOMS 2021 will be held on September 15-17, 2021, in Tainan, Taiwan. We expect that APNOMS 2021 will be even more successful. For more information, please visit .

\section{Appendix}

CORRECTION TO: A Collaborative DDoS Mitigation Solution Based on Ethereum Smart Contract and RNN LSTM. In: 2019 20th Asia-Pacific Network Operations and Management Symposium (APNOMS) (pp. 1-6). IEEE. DOI: https://doi.org/10. 34385/proc.56.TS9-2.

The authors were unaware that [1] had almost entirely plagiarized [2] and aim to grant full credit to the original authors.

[1] (RETRACTED ARTICLE) Dheeraj, J., Gurhubaran, S.: DDoS mitigation using blockchain. Int. J. Res. Eng. Sci. Manag. 1(10) (2018).

[2] (ORIGINAL ARTICLE) Rodrigues, B., Bocek, T., Lareida, A., Hausheer, D., Rafati, S., Stiller, B.: A blockchain-based architecture for collaborative DDoS mitigation with smart contracts. In: Tuncer, D., Koch, R., Badonnel, R., Stiller, B. (eds.) AIMS 2017. LNCS, vol. 10,356, pp. 16-29. Springer, Cham (2017). DOI: https:// doi.org/10.1007/978-3-319-60774-0_2.

Acknowledgements The authors would like to thank all APNOMS 2020 organizing committee members, including IEICE ICM and KICS KNOM members, for making this symposium successful. Our special thanks are extended to the IEEE ComSoc, DAEGU Convention \& Visitors Bureau (dcvb), Chunghwa Telecom, BcMon (Blockchain Monitoring System), BOSCO Technologies, and Ciena for their support.

\section{References}

1. Hong, J.W.: Toward global network management. J. Netw. Syst. Manag. 6(1), 91-93 (1998)

2. Ejiri, M., Park, J.T., Okazaki, H., Hong, J.W.: Managing the new telecommunications paradigms: a report on APNOMS 98. J. Netw. Syst. Manag. 6(4), 487-500 (1998)

3. Cho, Y.H., Tokunaga, H., Hong, J.W., Chujo, T.: Meeting the challenge in end-to-end service management: a report on APNOMS 99. J. Netw. Syst. Manag. 7(4), 495-498 (1999)

4. Taniguchi, T.: A report on APNOMS 2000. Glob. Commun. Newsl. IEEE Commun. Mag. 39(5), 1-4 (2001)

5. Chen, G., Caradharajan, V., Ray, P., Zuluaga, P.: Management for e-business in the new millennium. J. Netw. Syst. Manag. 10(2), 255-259 (2002) 
6. Kim, S., Suda, K., Hong, C.S., Kiriha, Y.: Integrated management for telecommunication solutions process, OSS and technology. J. Netw. Syst. Manag. 10(4), 531-535 (2002)

7. Mase, K., Ahn, I.S., Fujii, N., Shim, Y.C.: Managing pervasive computing and ubiquitous communications. J. Netw. Syst. Manag. 11(4), 505-509 (2003)

8. Fujii, N., Hong, J., Uno, H., Lee, K.-H.: Toward Managed Ubiquitous Information Society, APNOMS 2005 Report (2005). http://www.apnoms.org/reports/apnoms05-report.html

9. Hong, J., Kuriyama, H., Kim, Y.-T., Takano, M.: Management of convergence networks, services: a report on APNOMS 2006. J. Netw. Syst. Manag. 14(4), 603-608 (2006)

10. Kuriyama, H., Lee, K.-H., Kuo, G.S., Ata, S., Hong, C.S.: Managing next-generation networks, services: a report on APNOMS 2007. J. Netw. Syst. Manag. 16(1), 113-119 (2008)

11. Hong, J.W.-K., Meng, L., Kim, Y.-T., Uno, H., Ata, S., Ma, Y., Choi, D.: Challenges for next-generation network operations, service management: a report on APNOMS 2008. J. Netw. Syst. Manag. 17(1), 91-98 (2009)

12. Hong, J.W.K., Tu, Y.K., Hong, C.S., Tseng, S.S., Kiriha, Y., Chao, Y., Zhanikeev, Y., Song, W.C.: Managing clouds, smart networks and services: a report on APNOMS2011. J. Netw. Syst. Manag. 20(1), 134-142 (2012)

13. Ju, H., Hong, C.S., Takano, M., Yoo, J.H., Chang, K.Y., Yoshihara, K., Jeng, J.Y.: Management in the Big Data \& IoT era: a report on APNOMS 2012. J. Netw. Syst. Manag. 21(3), 517-524 (2013)

14. Kinoshita, K., Takano, M., Lee, Y.-W., Sun, S., Tonouchi, T., Kim, Y., Huang, T.-S.: Integrated management of network virtualization: a report on APNOMS 2013. J. Netw. Syst. Manag. 22(4), 509-516 (2014)

15. Kim, M.-S., Kamiyama, N., Yi, C.-W., Hong, W.-K., Tonouchi, T., Chen, C., Song, W.-C.: Managing a very connected world: a report on APNOMS 2015. J. Netw. Syst. Manag. 24(3), 754-763 (2016)

16. Kim, M.-S., Oishi, H., Huang, J.-L., Ju, H., Yoshihara, K., Yi, C.-W., Choi, T.: Managing a world of things: a report on APNOMS2017. J. Netw. Syst. Manag. 27(2), 541-549 (2018)

17. Kitaguchi, Y., Seok, W., Huang, J.L., Ju, H.T., Chen, Y.W., Yoshihara, K.: Management in a cyberphysical world: a report on APNOMS 2019. J. Netw. Syst. Manag. 28(2), 423-431 (2020)

Publisher's note Springer Nature remains neutral with regard to jurisdictional claims in published maps and institutional affiliations.

Seungjoon Seok received his B. S. degree in electronics engineering from Konkuk University and M.S. and Ph. D. degrees in communications engineering from Korea University, Seoul, South Korea, in 1997, 1999 and 2003, respectively. In 2003, he was a post doctor researcher at the University of Arkansas, Arkansas, USA. he worked Since March 2004, he has been a professor in the department of computer engineering, Kyunganam University. His research interests cover future Internet architecture, mobile Internet, edge computing, IoT platform, and smart system.

Yuncheng Zhu received his B.E. and M.E. degrees from Tsinghua University in 2007 and 2009, respectively, and his Ph.D. degree from the University of Tokyo in 2014. He has worked on performance measurement and optimization of mobile networks, Information-Centric Networking, Internet-of-Things, etc. He joined Google Japan in 2019 and currently is a Software Engineer there. He is a member of IEEE Communications Society and has served as an organizing committee member of APNOMS since 2016.

Jen-Jee Chen received the B.S. and M.S. degrees in computer science and information engineering from National Chiao Tung University, Hsinchu, Taiwan, in 2001 and 2003, respectively, and the Ph.D. degree in computer science from National Chiao Tung University, Hsinchu, Taiwan, in 2009. He was a Visiting Scholar at the University of Illinois, Urbana-Champaign, IL, USA, during the 2007-2008 academic year. Since 2019, he joined the College of Artificial Intelligence, National Chiao Tung University, Taiwan, where he is currently an Associate Professor. His research interests include AIoT, 5G wireless communications and networks, Robotics and Assistive Applications, and mobile computing.

Woojin Seok received a B.E, M.S.: and Ph.D. from Kyungpook National University, the University of North Carolina at Chapel Hill, U.S.A., and Chungnam National University, respectively. He is the 
KREONET center director in the Korea Institute of Science and Technology Information (KISTI). He is also an adjunct professor at the University of Science and Technology (UST). He is a committee member of KNOM and division chair of SDN/NFV forum PoC Division. His interesting research areas are Science DMZ, SDN/NFV, and satellite network

Kazuhiko Kinoshita received his B. E., M. E. and Ph. D degrees in information systems engineering from Osaka University, Osaka, Japan, in 1996, 1997 and 2003, respectively. From April 1998 to March 2002, he was an Assistant Professor at the Department of Information Systems Engineering, Graduate School of Engineering, Osaka University. From April 2002 to March 2008, he was an Assistant Professor at the Department of Information Networking, Graduate School of Information Science and Technology, Osaka University. From April 2008 to January 2015, he was an Associate Professor at the same University. Since February 2015, he has been a Professor at Tokushima University. His research interests include mobile networks, network management, and agent communications. Dr. Kinoshita is a member of IEEE and a senior member of IEICE.

Jiun-Long Huang received his B.S. and M.S. degrees in Computer Science and Information Engineering Department in National Chiao Tung University in 1997 and 1999, respectively, and his Ph.D. degree in Electrical Engineering Department in National Taiwan University in 2003. He joined National Chiao Tung University in 2005 and is currently a professor in Computer Science Department at National Chiao Tung University. His research interests include data mining, blockchain, and cloud computing.

Hongtaek Ju is a Professor in the Department of Computer Engineering, Keimyung University, Daegu, Republic of Korea. He received his B.S. degree in computer science from KAIST in 1989 and his M.S. and Ph.D. degree in the Department of Computer Science and Engineering from POSTECH respectively in 1991, 2002. He has worked on various research projects on network and system management. His research interests include network management architecture, traffic monitoring, and security management. He is a Member of IEEE Communications Society, KICS (The Korean Institute of Communications and Information Sciences), KSII (Korean Society for Internet Information), and KIPS (Korea Information Processing Society). He has served as an Organization Committee Member of APNOMS from 2005 and NOMS from 2010. He also has worked as a TPC Member of APNOMS, NOMS, and IM since 2006. He is an Associate Editor of the KNOM Review and International Journal of Network Management. 